\title{
REAL FUNCTIONS AND THE EXTENSION OF GENERALIZED PROBABILITY MEASURES II
}

\author{
JANA HAVLÍČKOVÁ
}

\begin{abstract}
We continue our study of the extensions of generalized probability measures. First, we describe some extensions of generalized random events (represented by classes of functions with values in $[0,1]$ ) to which generalized probability measures can be extended. Second, we study products of domains of probability and describe states on such products. Third, we show that the events in IF-probability, introduced by B. Riečan, form a suitable category isomorphic to a subcategory of the category of fuzzy random events. Consequently, IF-probability can be interpreted within fuzzy probability theory. We put forward some problems related to the extensions of probability domains and hint some applications.
\end{abstract}

\section{Introduction}

Following J. Novák 22]-24], using some recent results on generalized random events and states [2], 3], 20, [21, 30] and basic categorical methods [1], 15], in [19] we have studied the process of extending generalized probability measures.

In the second section, we present more results related to the extension process. The last two sections are devoted to the ralationships between IF-probability, introduced by B. R i ěc a $\mathrm{n}$ in [31], and fuzzy probability [8], 18], 34].

Relevant notions concerning generalized random events and generalized probability measures (states) are recalled in the text. Further, if $\mathbf{A}$ is a $\sigma$-field of sets, then $\mathcal{M}(\mathbf{A})$ denotes the class of all $\mathbf{A}$-measurable functions whose values are in $[0,1]$. As usually, $\exists$ ! means "there exists a unique".

(C) 2015 Mathematical Institute, Slovak Academy of Sciences.

2010 Mathematics Subject Classification: 54C20, 28A05, 60A86, 28C99, 06F99.

Keywords: D-poset of fuzzy sets, sequentially continuous D-homomorphism, extension, sober, sobrification, product, bold algebra, Łukasiewicz tribe, epireflective subcategory, fuzzy probability, IF-probability.

This work was supported by the Slovak Scientific Grant Agency VEGA project 1/0330/13. 
D-posets have been introduced in 20] in order to model events in quantum probability. They generalize Boolean algebras, MV-algebras and other probability domains, and provide a category in which observables and states become morphisms [2], 11. Recall that a D-poset is a partially ordered set with the greatest element $1_{X}$, the least element $0_{X}$, and a partial binary operation called difference, such that $a \ominus b$ is defined iff $b \leq a$, and the following axioms are assumed:

(D1) $a \ominus 0_{X}=a$ for each $a \in X$;

(D2) If $c \leq b \leq a$, then $a \ominus b \leq a \ominus c$ and $(a \ominus c) \ominus(a \ominus b)=b \ominus c$.

A canonical example is the interval $[0,1]=I$ (linearly ordered, $a \ominus b=a-b$ whenever $b \leq a$ ). D-posets of fuzzy sets are fundamental to applications [16], 17], i.e., systems $\mathcal{X} \subseteq I^{X}$ carrying the coordinatewise partial order, coordinatewise convergence of sequences, containing the top and bottom elements of $I^{X}$, and closed with respect to the partial operation difference defined coordinatewise. We always assume that $\mathcal{X}$ is reduced, i.e., for $x, y \in X, x \neq y$, there exists $u \in \mathcal{X}$ such that $u(x) \neq u(y)$. Denote ID the category having (reduced) D-posets of fuzzy sets as objects and having sequentially continuous D-homomorphisms (preserving constants, order, and the difference) as morphisms. Objects of ID are subobjects of the powers $I^{X}$.

\section{Extensions}

Extensions of D-posets of fuzzy sets have been studied in [9], 11] 13, [19, [26]. Essentially, there are two types of extensions. First, we can add functions, and then, we speak of an ID-extension (cf. [19]). Second, we can extend the domain of functions and such extensions have stochastic applications, e.g., when studying the duality between generalized random variables and observables [5], 7], 8, 10, [14]-16, 26].

Let $\mathcal{X} \subseteq I^{X}$ be a D-poset of fuzzy sets. Denote $\mathcal{S}(\mathcal{X})$ the set of all sequentially continuous D-homomorphisms of $\mathcal{X}$ into $I$; the elements of $\mathcal{S}(\mathcal{X})$ are called states. In what follows, each $x \in X$ will be considered as the evaluation state on $\mathcal{X}: x(u)=u(x), u \in \mathcal{X}$. If $X=\mathcal{S}(\mathcal{X})$, then $\mathcal{X}$ is said to be sober (cf. [26]). Let $Y$ be a set of states such that $X \subseteq Y \subseteq \mathcal{S}(\mathcal{X})$. For $u \in \mathcal{X}$, put $e v_{Y}(u)=\{y(u) ; y \in Y\} \in I^{Y}$ and by $e v_{Y}$ denote the corresponding map of $\mathcal{X}$ into $I^{Y}$. Put $\mathcal{X}_{Y}=\left\{e v_{Y}(u) ; u \in \mathcal{X}\right\}$. A trivial proof of the next lemma is omitted.

\section{LEMMA 2.1.}

(i) $\mathcal{X}_{Y}$ is a D-poset of fuzzy sets (with respect to the D-poset structure inherited from $\left.I^{Y}\right)$,

(ii) $e v_{Y}$ is an isomorphism. 
Definition 2.2. Let $\mathcal{X} \subseteq I^{X}$ be a D-poset of fuzzy sets and let $Y$ be a set of states such that $X \subseteq Y \subseteq \mathcal{S}(\mathcal{X})$. Then, $\mathcal{X}_{Y}$ is said to be a domain extension of $\mathcal{X}$. If $Y=\mathcal{S}(\mathcal{X})$, then $\mathcal{X}_{Y}$ is said to be the sobrification of $\mathcal{X}$.

In accordance with [26, for $\mathcal{X} \subseteq I^{X}$ and $Y=\mathcal{S}(\mathcal{X})$, the sobrification $\mathcal{X}_{Y}$ of $\mathcal{X}$ will be denoted by $\mathcal{X}^{*}$. Denote SID the (full) subcategory of ID consisting of sober D-posets of fuzzy sets.

\section{LEMMA 2.3.}

(i) Let $\mathcal{X} \subseteq I^{X}$ be a $\mathrm{D}$-poset of fuzzy sets and let $h$ be a sequentially continuous $\mathrm{D}$-homomorphism of $\mathcal{X}$ into a sober $\mathrm{D}$-poset of fuzzy sets $\mathcal{Z}$. Then, there exists a unique sequentially continuous $\mathrm{D}$-homomorphism $h^{*}$ of $\mathcal{X}^{*}=\mathcal{X}_{\mathcal{S}(\mathcal{X})}$ into $\mathcal{Z}$ such that $h=h^{*} \circ e v_{\mathcal{S}}(\mathcal{X})$.

(ii) Let $h$ be a sequentially continuous D-homomorphism of a D-poset of fuzzy sets $\mathcal{X}$ into a D-poset of fuzzy sets $\mathcal{Y}$. Then, there exists a unique sequentially continuous D-homomorphism $h^{*}$ of $\mathcal{X}^{*}$ into $\mathcal{Y}^{*}$ such that $h^{*} \circ e v_{\mathcal{S}(\mathcal{X})}=h \circ e v_{\mathcal{S}(\mathcal{Y})}$.

Proof. (i) By the previous lemma, $\mathcal{X}$ and $\mathcal{X}^{*}$ are isomorphic. Define $h^{*}$ by putting $h^{*}\left(u^{*}\right)=h(u), u \in \mathcal{X}$. Clearly, $h^{*}$ is a sequentially continuous Dhomomorphism $h^{*}$ of $\mathcal{X}^{*}$ into $\mathcal{Z}$. We have to prove the uniqueness of $h^{*}$. So, let $g$ be a sequentially continuous D-homomorphism of $\mathcal{X}^{*}$ into $\mathcal{Z}$ such that $h=g \circ e v_{\mathcal{S}(\mathcal{X})}$. Then

$$
g\left(u^{*}\right)=h(u)=h^{*}\left(u^{*}\right) \text { for all } u^{*} \in \mathcal{X}^{*} .
$$

Hence $g=h^{*}$ and (i) follows.

(ii) Follows from (i).

TheOREM 2.4. The subcategory SID of ID is epireflective in ID.

P r o of. The assertion follows from the pre previous lemma.

Note that the sobrification sending a D-poset of fuzzy sets $\mathcal{X}$ to $\mathcal{X}^{*}$ yields a functor (epireflector) sending a morphism $h: \mathcal{X} \longrightarrow \mathcal{Y}$ to $h^{*}: \mathcal{X}^{*} \longrightarrow \mathcal{Y}^{*}$.

Example 2.5. For $X=\{0,1\}$, let $\mathcal{X} \subseteq I^{X}$ be the $\sigma$-field of all subsets of $X$. The set $\mathcal{S}(\mathcal{X})$ of all states of $\mathcal{X}$ is the same as the set of all probability measures on the $\sigma$-field in question [26]. Each $s \in \mathcal{S}(\mathcal{X})$ can be visualized as an element $a_{s}$ of the closed unit interval $[0,1]$, where the number $a_{s}$ is equal to the corresponding probability of the singleton $\{0\}$ and $\left(1-a_{s}\right)$ is equal to the corresponding probability of the singleton $\{1\}$; we identify $s$ and the corresponding number $a_{s}$ and, consequently, we identify $\mathcal{S}(\mathcal{X})$ and $[0,1]$. Further, the sobrification $\mathcal{X}^{*}$

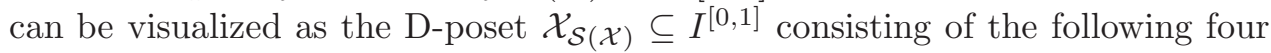
functions: constant functions $0_{[0,1]}$ and $1_{[0,1]}$, functions $x$ and $1-x, x \in[0,1]$. 
Now, we turn to ID-extensions. Even though the embedding of a field of sets $\mathbf{A}$ into the generated $\sigma$-field $\sigma(\mathbf{A})$ is well-known, there are still some unanswered related questions. We present some motivating examples and, in order to describe additional properties of extensions, we introduce some new notions.

Let $\mathcal{X} \subseteq I^{X}$ be a D-poset of fuzzy sets and let $\mathcal{Y} \subseteq I^{X}$ be an ID-extension of $\mathcal{X}$. Recall (cf. [19]) that if each state on $\mathcal{X}$ can be extended to a state on $\mathcal{Y}$, i.e., $(\forall s \in \mathcal{S}(\mathcal{X}))(\exists t \in \mathcal{S}(\mathcal{Y}))[t \mid \mathcal{X}=s]$, then $\mathcal{X}$ is said to be $\mathcal{S}(\mathcal{X})$-embedded in $\mathcal{Y}$.

Let $\mathcal{X} \subseteq I^{X}$ be a D-poset of fuzzy sets and let $\mathcal{Y} \subseteq I^{X}$ be an ID-extension of $\mathcal{X}$. Let $t$ be a state on $\mathcal{Y}$ and let $t \uparrow \mathcal{X}$ be the restriction of $t$ to $\mathcal{X}$. Since $t\lceil\mathcal{X} \in \mathcal{S}(\mathcal{X})$, the restriction yields a restriction map $r$ of $\mathcal{S}(\mathcal{Y})$ into $\mathcal{S}(\mathcal{X})$ sending $t$ to $r(t)=t\lceil\mathcal{X}$.

Definition 2.6. Let $\mathcal{X} \subseteq I^{X}$ be a D-poset of fuzzy sets and let $\mathcal{Y} \subseteq I^{X}$ be an ID-extension of $\mathcal{X}$. If the restriction map $r: \mathcal{S}(\mathcal{Y}) \longrightarrow \mathcal{S}(\mathcal{X})$ is one-to-one and onto, then $\mathcal{Y}$ is said to be a state extension of $\mathcal{X}$. A state extension $\mathcal{Y}$ of $\mathcal{X}$ is said to be maximal if there is no proper state extension $\mathcal{Z} \subseteq I^{X}$ of $\mathcal{Y}$. Let $\mathcal{C}$ be a class of state extensions of $\mathcal{X}$ and let $\mathcal{Y} \in \mathcal{C}$. If there is no proper extension of $\mathcal{X}$ in $\mathcal{C}$, then $\mathcal{Y}$ is said to be maximal in $\mathcal{C}$. If $\mathcal{Y}$ is a state extension of $\mathcal{X}$ and $\mathcal{Y}$ is an ID-extension of each state extension $\mathcal{Z}$ of $\mathcal{X}$, then $\mathcal{Y}$ is said to be the absolute state extension of $\mathcal{X}$. If $\mathcal{Y} \in \mathcal{C}$ and $\mathcal{Y}$ is an ID-extension of each state extension $\mathcal{Z}$ of $\mathcal{X}$ in $\mathcal{C}$, then $\mathcal{Y}$ is said to be the absolute state extension of $\mathcal{X}$ in $\mathcal{C}$.

Lemma 2.7. Let $\mathcal{X} \subseteq I^{X}$ be a D-poset of fuzzy sets and let $\mathcal{Y} \subseteq I^{X}$ be an ID-extension of $\mathcal{X}$. The following are equivalent:

(i) $\mathcal{Y}$ is a state extension of $\mathcal{X}$;

(ii) $(\forall s \in \mathcal{S}(\mathcal{X}))(\exists ! t \in \mathcal{S}(\mathcal{Y}))[t \uparrow \mathcal{X}=s]$;

(iii) $(\forall t \in \mathcal{S}(\mathcal{Y}))(\exists ! s \in \mathcal{S}(\mathcal{X}))[t\lceil\mathcal{X}=s]$.

Pr o of. The proof is straightforward and is left out.

ExAmple 2.8. Consider $\mathcal{X}$ and its sobrification $\mathcal{X}^{*} \subseteq I^{[0,1]}$ in the previous example. Let $\mathbf{B}$ be the $\sigma$-field of all Borel measurable subsets of $[0,1]$ and let $\mathcal{M}(\mathbf{B})$ be the set of all $\mathbf{B}$-measurable functions with values in I. Then, $\mathcal{M}(\mathbf{B})$ can be considered as an ID-extension of $\mathcal{X}^{*}$. Clearly, $\mathcal{X}^{*}$ is $\mathcal{S}\left(\mathcal{X}^{*}\right)$-embedded in $\mathcal{S}(\mathcal{M}(\mathbf{B}))$. On the other hand, the restriction map $r$ of $\mathcal{S}(\mathcal{M}(\mathbf{B}))$ into $\mathcal{S}\left(\mathcal{X}^{*}\right)$ is far from being one-to-one. Hence, $\mathcal{M}(\mathbf{B})$ fails to be a state extension of $\mathcal{X}^{*}$.

Let $X$ be a set, let $\mathbf{A}$ be a field of subsets of $X$, let $\sigma(\mathbf{A})$ be the generated $\sigma$-field, and let $\mathbf{M}_{\mathbf{A}}$ be the $\sigma$-field of all absolutely $\mathbf{A}$-measurable subsets of $X$ ( $M \subseteq X$ belongs to $\mathbf{M}_{\mathbf{A}}$ if and only if it is $p$-measurable for all $p \in \mathcal{P}(\mathbf{A})$, see [19]). Both $\sigma(\mathbf{A})$ and $\mathbf{M}_{\mathbf{A}}$ can be considered as ID-extensions of $\mathbf{A}$. A natural question arises what can be said about such extensions. 
Example 2.9. Let $\mathbf{A}$ be a field of subsets of $X$, let $\sigma(\mathbf{A})$ be the generated $\sigma$-field, and let $\mathbf{P}(X)$ be the $\sigma$-field of all subsets of $X$. For a system $\mathbf{B}$ of subsets of $X$, define $\operatorname{cl}(\mathbf{B}) \subseteq \mathbf{P}(X)$ as the set of all sequential limits of sequences of sets in $\mathbf{B}$ and, inductively, for each ordinal number $\alpha \leq \omega_{1}$ define $\operatorname{cl}^{0}(\mathbf{B})=\mathbf{B}, \operatorname{cl}^{\alpha}(\mathbf{B})=$ $\mathrm{cl}\left(\mathrm{cl}^{\alpha-1}(\mathbf{B})\right)$ for an isolated ordinal number, $\mathrm{cl}^{\alpha}(\mathbf{B})=\mathrm{cl}\left(\bigcup_{\beta<\alpha} \mathrm{cl}^{\beta}(\mathbf{B})\right)$ for a limit ordinal number; it is known (cf. 25]) that each $\mathrm{cl}^{\alpha}$ is a closure operator (need not be idempotent), $\mathrm{cl}^{\omega_{1}}$ is a topological closure operator (topology), each $\mathrm{cl}^{\alpha}(\mathbf{A})$ is a field of subsets of $X$, and $\mathrm{cl}^{\omega_{1}}(\mathbf{A})=\sigma(\mathbf{A})$. If $\mathrm{cl}^{\alpha}(\mathbf{B})=\mathbf{C}$ for some ordinal number $\alpha$, then $\mathbf{B}$ is said to be sequentially dense in $\mathbf{C}$. For each ordinal number $\alpha \leq \omega_{1}$, put $\mathrm{cl}^{\alpha}(\mathbf{A})=\mathbf{A}_{\alpha}$. Then (see in [19, Example 4.2]), each $\mathbf{A}_{\alpha}$ is a state extension of $\mathbf{A}$. For $\mathcal{C}=\left\{\mathbf{A}_{\alpha} ; \alpha \leq \omega_{1}\right\}, \mathbf{A}_{\omega_{1}}=\sigma(\mathbf{A})$ is the absolute state extension of $\mathbf{A}$ in $\mathcal{C}$.

Example 2.10. Let $\mathbf{A}$ be a field of subsets of $X$ and let $\mathbf{M}_{\mathbf{A}}$ be the $\sigma$-field of all absolutely A-measurable subsets of $X$. In [19, Example 4.3], we have erroneously claimed that " $\mathbf{M}_{\mathbf{A}}$ is the largest $\sigma$-field of subsets of $X$ in which A is $\mathcal{P}(\mathbf{A})$-embedded". For sure, $\mathbf{M}_{\mathbf{A}}$ is the largest $\sigma$-field of subsets of $X$ to which each probability measure on $\mathbf{A}$ has a measurable extension. Hence, $\mathcal{P}(\mathbf{A})$-embeddedness has to be replaced by a more restrictive assumption.

Problem 1. Let $\mathbf{A}$ be a field of subsets of $X$ and let $\mathbf{M}_{\mathbf{A}}$ be the $\sigma$-field of all absolutely $\mathbf{A}$-measurable subsets of $X$. Is $\mathbf{M}_{\mathbf{A}}$ a state extension of $\mathbf{A}$ ?

Remark 2.11. Observe that a positive solution to Problem 1 implies that the extension $\mathbf{A} \hookrightarrow \mathbf{M}_{\mathbf{A}}$ yields an epireflection. Indeed, if each probability measure on $\mathbf{A}$ can be uniquely extended to a probability measure on $\mathbf{M}_{\mathbf{A}}$, then the corresponding epireflector can be constructed by standard categorical methods.

Problem 2. Characterize fields of sets $\mathbf{A}$ for which $\mathbf{M}_{\mathbf{A}}$ is a state extension.

Problem 3. Let $\mathcal{X} \subseteq I^{X}$ be a D-poset of fuzzy sets. Can $\mathcal{X}$ have different maximal state extensions?

Problem 4. Characterize D-posets of fuzzy sets having state extensions (absolute state extensions, or absolute state extensions in a nontrivial class of state extensions).

Since, in generalized probability theory, bold algebras provide a natural model of generalized random events, we will study ID-extensions which are bold algebras.

Recall [3] that a bold algebra is a system $\mathcal{X} \subseteq[0,1]^{X}$ containing the constant functions $0_{X}, 1_{X}$ and closed with respect to the usual (Lukasiewicz) operations: for $u, v \in \mathcal{X}$ put

$$
(u \oplus v)(x)=u(x) \oplus v(x)=\min \{1, u(x)+v(x)\}, \quad u^{*}(x)=1-u(x), \quad x \in X .
$$


Bold algebras are MV-algebras representable as $[0,1]$-valued functions, MV-algebras generalize Boolean algebras and bold algebras generalize in a natural way fields of sets (viewed as indicator functions). More information concerning MV-algebras and probability on MV-algebras can be found in [29. If a bold algebra $\mathcal{X} \subseteq[0,1]^{X}$ is sequentially closed in $[0,1]^{X}$ (with respect to the coordinatewise sequential convergence), then $\mathcal{X}$ is a Eukasiewicz tribe $(\mathcal{X}$ is closed not only with respect to finite, but also with respect to countable Eukasiewicz sums, cf. [6, Corollary 2.8]). It is known (cf. [3]) that if $\mathcal{X} \subseteq[0,1]^{X}$ is a Eukasiewicz tribe, then there exists a unique $\sigma$-field $\mathbf{A}_{\mathcal{X}}$ of subsets of $X$ such that $\mathbf{A}_{\mathcal{X}} \subseteq \mathcal{X} \subseteq \mathcal{M}\left(\mathbf{A}_{\mathcal{X}}\right)$ and $\mathcal{X}=\mathcal{M}\left(\mathbf{A}_{\mathcal{X}}\right)$ if and only if $\mathcal{X}$ contains all constant functions $r_{X}, r \in[0,1]$. Let $\mathcal{X} \subseteq[0,1]^{X}$ be a bold algebra. Then, $[0,1]^{X}$ is a Eukasiewicz tribe containing $\mathcal{X}$ and the intersection of all Eukasiewicz tribes $\mathcal{Y} \subseteq[0,1]^{X}$ such that $\mathcal{X} \subseteq \mathcal{Y}$ is a Eukasiewicz tribe; it will be denoted by $\sigma(\mathcal{X})$. Eukasiewicz tribes of the form $\left.\mathcal{M}\left(\mathbf{A}_{\sigma(\mathcal{X}}\right)\right)$ are called generated. Each bold algebra $\mathcal{X} \subseteq[0,1]^{X}$ is a lattice where, for $u, v \in \mathcal{X}$, we have $(u \vee v)(x)=u(x) \vee v(x),(u \wedge v)(x)=u(x) \wedge v(x), x \in X$, and each ID-poset $\mathcal{X} \subseteq[0,1]^{X}$ which is a lattice (with respect to the coordinatewise order) can be reorganized into a bold algebra (the Łukasiewicz operations can be redefined via the difference and order, cf. [16, [28]). Finally, each bold algebra can be considered as an object of ID. The corresponding subcategory is denoted by BID and CGBID denotes the subcategory of generated Łukasiewicz tribes.

Let $\mathcal{X} \subseteq I^{X}$ be a D-poset of fuzzy sets. Clearly, the intersection of all bold algebras $\mathcal{Y} \subseteq I^{X}$ such that $\mathcal{Y}$ is an ID-extension of $\mathcal{X}$ is the smallest ID-extension of $\mathcal{X}$ which is a bold algebra; denote it by $b(\mathcal{X})$. Below we also give an inductive construction of $b(\mathcal{X})$.

For $u, v \in I^{X}$, define pointwise binary operations $u \vee_{p} v$ and $u \wedge_{p} v$ as follows: $\left(u \vee_{p} v\right)(x)=u(x) \vee v(x),\left(u \wedge_{p} v\right)(x)=u(x) \wedge v(x), x \in X$. For $u, v \in I^{X}, v \leq u$, define pointwise a partial operation $u-v:(u-v)(x)=u(x)-v(x), x \in X$.

For $n=0,1,2, \ldots$, define $b_{0}(\mathcal{X})=\mathcal{X}, b_{n}(\mathcal{X})=\left\{u \vee_{p} v ; u, v \in b_{n-1}(\mathcal{X})\right\}$ $\cup\left\{u \wedge_{p} v ; u, v \in b_{n-1}(\mathcal{X})\right\}$ for $n$ odd, $b_{n}(\mathcal{X})=\left\{u-v ; u, v \in b_{n-1}(\mathcal{X}), v \leq u\right\}$ for $n$ even.

LEMMA 2.12. $b(\mathcal{X})=\bigcup_{n=0}^{\infty} b_{n}(\mathcal{X})$.

P r o o f. It is easy to see that $\bigcup_{n=0}^{\infty} b_{n}(\mathcal{X}) \subseteq I^{X}$ contains the constant functions $0_{X}, 1_{X}$ and it is closed with respect to $u \vee_{p} v, u \wedge_{p} v$ and the partial difference. Indeed, if $u, v \in b(\mathcal{X})$, then there exists a natural number $n$ such that $u, v \in b_{n}(\mathcal{X})$. Hence, $\left(u \vee_{p} v\right),\left(u \wedge_{p} v\right) \in b_{n+2}(\mathcal{X})$. If $v \leq u$, then also $(u-v) \in$ $b_{n+2}(\mathcal{X})$. Thus, $\bigcup_{n=0}^{\infty} b_{n}(\mathcal{X})$ is a bold algebra. Let $\mathcal{Y} \subseteq I^{X}$ be an ID-extension of $\mathcal{X}$ and assume that $\mathcal{Y}$ is a bold algebra. Then, $\mathcal{Y}$ contains the constant functions $0_{X}, 1_{X}$ and it is closed with respect to $u \vee_{p} v, u \wedge_{p} v$ and the partial difference. Thus, $\bigcup_{n=0}^{\infty} b_{n}(\mathcal{X}) \subseteq \mathcal{Y}$ and the proof is complete. 
Lemma 2.13. Let $\mathcal{X} \subseteq I^{X}$ be a sober $\mathrm{D}$-poset of fuzzy sets. Then, $\mathcal{X}$ is $\mathcal{S}(\mathcal{X})$ -embedded in $b(\mathcal{X})$.

P r o of. Let $s$ be a state on $\mathcal{X}$. Since $\mathcal{X}$ is sober, there exists a unique $x \in X$ such that $s$ is the evaluation state $s_{x}$. Then the $x$ th projection $p r_{x}$ of $I^{X}$ to the $x$ th factor $I_{x}$ restricted to $\mathcal{X}$ is equal to $s$ and the restriction $p r_{x} \uparrow b(\mathcal{X})$ is a state on $b(\mathcal{X})$ which extends $s$.

\section{Products}

The random events in IF-probability 31] are pairs $(u, v)$ of fuzzy random events, i.e., elements of probability domains of the form $\mathcal{M}(\mathbf{A}) \times \mathcal{M}(\mathbf{A})$ such that $u \leq v$, and some relevant constructions in the IF-probability can be carried out (cf. [31], 32]) as the corresponding constructions in fuzzy probability theory (via $\mathcal{M}(\mathbf{A}) \times \mathcal{M}(\mathbf{A}))$. This section is devoted to products of D-posets of fuzzy sets and some of the results will be used in the next section.

Let $\mathcal{X} \subseteq I^{X}$ and $\mathcal{Y} \subseteq I^{Y}$ be D-posets of fuzzy sets. Recall that (see [26], 27]), along with the two natural projections, the product $\mathcal{X} \times \mathcal{Y}$ consists of all pairs $(u, v), u \in \mathcal{X}, v \in \mathcal{Y}$, where the ID-structure (partial order, difference, and convergence) is defined coordinatewise; observe that if $Z$ is the disjoint union of $X$ and $Y$ (their coproduct in the category of sets and maps) then each $(u, v)$ can be visualized as a function $w$ on $Z$, where $u$ and $v$ are "disjoinly glued" to form $w$. Let $T$ be a (nonempty) set and let $\left\{\mathcal{X}_{t} ; t \in T\right\}$ be an indexed family of D-posets of fuzzy sets. As before, their product $\prod_{t \in T} \mathcal{X}_{t}$, along with the indexed family $\left\{p r_{t} ; t \in T\right\}$ of natural projections $p r_{s}: \prod_{t \in T} \mathcal{X}_{t} \longrightarrow \mathcal{X}_{s}$, $s \in T$, consists of the indexed family $\left\{u_{t} ; u_{t} \in \mathcal{X}_{t}, t \in T\right\}$, equipped with the coordinatewise ID-structure.

Further, if $\mathcal{X}_{t}, t \in T$, are bold algebras, then the Eukasiewicz operations $\oplus, \odot,(\cdot)^{c}$ on their product are defined pointwise and $\prod_{t \in T} \mathcal{X}_{t}$ becomes the product bold algebra.

Let $n>1$ be a natural number. Denote $\mathbf{B I D}_{n}$ the category of products bold algebras of the form $\prod_{i=1}^{n} \mathcal{X}_{i}$ as objects and $n$-tuples of sequentially continuous D-homomorphisms as morphisms, i.e., if $h_{i}: \mathcal{X}_{i} \longrightarrow \mathcal{Y}_{i}, i=1,2, \ldots, n$, are sequentially continuous D-homomorphisms, then $\left(h_{1}, h_{2}, \ldots, h_{n}\right)$ is the corresponding morphism on $\prod_{i=1}^{n} \mathcal{X}_{i}$ into $\prod_{i=1}^{n} \mathcal{Y}_{i}$ in $\mathbf{B I D}_{n}$ and the composition of two $n$-tuples $\left(h_{1}, h_{2}, \ldots, h_{n}\right)$ and $\left(g_{1}, g_{2}, \ldots, g_{n}\right)$ is defined coordinatewise, i.e., if the compositions $g_{i} \circ h_{i}, i=1,2, \ldots, n$, are defined, then $\left(g_{1}, g_{2}, \ldots, g_{n}\right) \circ$ $\left(h_{1}, h_{2}, \ldots, h_{n}\right)=\left(g_{1} \circ h_{1}, g_{2} \circ h_{2}, \ldots, g_{n} \circ h_{n}\right)$. Recall that a generated Łukasiewicz tribe is a bold algebra $\mathcal{M}(\mathbf{A})$ of all measurable $[0,1]$-valued functions with respect to a $\sigma$-algebra $\mathbf{A}$ of subsets. Denote $\mathbf{C G B I D}_{n}$ the (full) subcategory of $\mathbf{B I D}_{n}$ 
the object of which are the products of generated Eukasiewicz tribes of the form $\prod_{i=1}^{n} \mathcal{M}\left(\mathbf{A}_{i}\right)$.

Let $n>1$ be a natural number, let $\mathcal{X}_{i}, i=1,2, \ldots, n$, be D-posets of fuzzy sets, and let $\mathcal{X}=\prod_{i=1}^{n} \mathcal{X}_{i}$ be their product. Recall that a sequentially continuous D-homomorphism on $\mathcal{X}$ into $I$ is said to be a state. In this section, we generalize some results on states on products in 33 .

Let $n>1$ be a natural number, let $\mathcal{X}_{i}, i=1,2, \ldots, n$, be D-posets of fuzzy sets, and let $\mathcal{X}=\prod_{i=1}^{n} \mathcal{X}_{i}$ be their product. Let $h_{i}, i=1,2, \ldots, n$, be a sequentially continuous D-homomorphism of $\mathcal{X}_{i}$ into a generated Eukasiewicz tribe $\mathcal{M}(\mathbf{A}) \subseteq I^{Y}$, and let $w_{i} \in \mathcal{M}(\mathbf{A}), i=1,2, \ldots, n$, be functions such that $\sum_{i=1}^{n} w_{i}(y)=1$ for all $y \in Y$. For $u=\left(u_{1}, \ldots, u_{n}\right) \in \mathcal{X}$, put $h\left(u_{1}, \ldots, u_{n}\right)=$ $\sum_{i=1}^{n} w_{i} h_{i}\left(u_{i}\right)$; clearly, $h\left(u_{1}, \ldots, u_{n}\right) \in \mathcal{M}(\mathbf{A})$. Denote $h=\sum_{i=1}^{n} w_{i} h_{i}$ the resulting map of $\mathcal{X}$ into $\mathcal{M}(\mathbf{A})$.

The proof of the next lemma is straightforward and is omitted.

Lemma 3.1. The map $h$ is a sequentially continuous D-homomorphism of $\mathcal{X}$ into $\mathcal{M}(\mathbf{A})$.

Definition 3.2. Let $n>1$ be a natural number, let $\mathcal{X}_{i}, i=1,2, \ldots, n$, be D-posets of fuzzy sets, and let $\mathcal{X}$ be their product. Let $h_{i}, i=1,2, \ldots, n$, be a sequentially continuous D-homomorphism of $\mathcal{X}_{i}$ into a generated Eukasiewicz tribe $\mathcal{M}(\mathbf{A}) \subseteq I^{Y}$, and let $w_{i} \in \mathcal{M}(\mathbf{A}), i=1,2, \ldots, n$, be functions such that $\sum_{i=1}^{n} w_{i}(y)=1$ for all $y \in Y$. Then, $h=\sum_{i=1}^{n} w_{i} h_{i}$ is said to be a convex combination of $h_{i}, i=1,2, \ldots, n$.

Let $\{a\}$ be a singleton and let $\mathbf{T}=\{\emptyset,\{a\}\}$ be the trivial $\sigma$-field of subsets of $\{a\}$. As usual, we identify $I$ and $\mathcal{M}(\mathbf{T})$. Observe that the convex combination of sequentially continuous D-homomorphisms into $I=\mathcal{M}(\mathbf{T})$ is a state.

Definition 3.3. Let $n>1$ be a natural number, let $\mathcal{X}_{i}, i=1,2, \ldots, n$, be D-posets of fuzzy sets, and let $\mathcal{X}$ be their product. Let $h$ be a sequentially continuous D-homomorphism of $\mathcal{X}$ into a generated Łukasiewicz tribe $\mathcal{M}(\mathbf{A}) \subseteq Y$. For $i \in\{1,2, \ldots, n\}$, if $h\left(0_{1}, \ldots, 0_{i-1}, 1_{i}, 0_{i+1}, \ldots, 0_{n}\right)=w$, and $w(y)>0$ for all $y \in Y$, then we say that $h$ strongly depends on the $i$ th coordinate.

TheOREM 3.4. Let $n>1$ be a natural number, let $\mathcal{X}_{i}, i=1,2, \ldots, n$, be D-posets of fuzzy sets, let $\mathcal{X}$ be their product, and let $h$ be a sequentially continuous D-homomorphism of $\mathcal{X}$ into a generated Eukasiewicz tribe $\mathcal{M}(\mathbf{A}) \subseteq I^{Y}$ which strongly depends on all coordinates. Then, there are unique sequentially continuous D-homomorphisms $h_{i}: \mathcal{X}_{i} \longrightarrow \mathcal{M}(\mathbf{A})$, and unique functions $w_{i} \in \mathcal{M}(\mathbf{A})$ such that $\sum_{i=1}^{n} w_{i}(y)=1$ for all $y \in Y, i=1,2, \ldots, n$, and $h=\sum_{i=1}^{n} w_{i} h_{i}$. 
Proof. For $i \in\{1,2, \ldots, n\}$, put $w_{i}=h\left(0_{1}, \ldots, 0_{i-1}, 1_{i}, 0_{i+1}, \ldots, 0_{n}\right)$. Then $0<w_{i}(y)$ and $1=h\left(1_{1}, 1_{2}, \ldots, 1_{n}\right)(y)=\sum_{i=1}^{n} h\left(0_{1}, \ldots, 0_{i-1}, 1_{i}, 0_{i+1}, \ldots, 0_{n}\right)(y)$ for all $y \in Y, i \in\{1,2, \ldots, n\}$. Clearly, for each $i \in\{1,2, \ldots, n\}$,

$$
h_{i}(u)=h\left(0_{1}, \ldots, 0_{i-1}, u, 0_{i+1}, \ldots, 0_{n}\right) / w_{i}, \quad u \in \mathcal{X}_{i},
$$

defines a sequentially continuous D-homomorphism $h_{i}: \mathcal{X}_{i} \longrightarrow \mathcal{M}(\mathbf{A})$. Further, we have to prove

$$
h\left(u_{1}, \ldots, u_{n}\right)=\sum_{i=1}^{n} w_{i} h_{i}\left(u_{i}\right), \quad \text { for all }\left(u_{1}, \ldots, u_{n}\right) \in \prod_{i=1}^{n} \mathcal{X}_{i} .
$$

It is easy to see that

$$
h\left(u_{1}, \ldots, u_{n}\right)=\sum_{i=1}^{n} w_{i} h\left(0_{1}, \ldots, 0_{i-1}, u_{i}, 0_{i+1}, \ldots, 0_{n}\right) / w_{i}
$$

and the assertion follows. An analogous argument shows that if $h=\sum_{i=1}^{n} v_{i} g_{i}$, then $w_{i}=v_{i}$, and $h_{i}=g_{i}$ for all $i \in\{1,2, \ldots, n\}$.

Observe that Theorem 3.4 generalizes Theorem 3.5 in 33 . Indeed, if $\mathbf{A}$ is the trivial $\sigma$-field of subsets of a singleton, then $\mathcal{M}(\mathbf{A})=I$, the homomorphism $h$ into $\mathcal{M}(\mathbf{A})$ becomes a state, and the strong dependence of $h$ amounts to the dependence of $h$.

In [13, it is proved that CGBID is an epireflective subcategory of BID. This is a positive solution to Problem 2 posed in [19]. Let $\mathcal{X} \subseteq I^{X}$ be a bold algebra. Then, $\mathcal{M}\left(\mathbf{A}_{\sigma(\mathcal{X})}\right)$ is its epireflection. In connection with products of probability domains, it is natural to ask whether the epireflection is productive. In general topology, the question whether an epireflector like the Čech-Stone compactification, the Hewitt realcompactification, etc. is productive, is rather hard. For sequential envelopes, it is proved [4] that the corresponding epireflector is productive. We have proved that the reflector sending $\mathcal{X}$ to $\mathcal{M}\left(\mathbf{A}_{\sigma(\mathcal{X})}\right)$ is productive, too.

Theorem 3.5. Let $\mathcal{X} \subseteq I^{X}, \mathcal{Y} \subseteq I^{Y}$ be bold algebras and let $\mathcal{X} \times \mathcal{Y}$ be their product. Then, $\mathcal{M}\left(\mathbf{A}_{\sigma(\mathcal{X})}\right) \times \mathcal{M}\left(\mathbf{A}_{\sigma(\mathcal{Y})}\right)$ is the epireflection of $\mathcal{X} \times \mathcal{Y}$ into the subcategory CGBID of BID.

P r o of. Let $\mathbf{A}$ be a $\sigma$-field of sets and let $h$ be a sequentially continuous D-homomorphism on $\mathcal{X} \times \mathcal{Y}$ into $\mathcal{M}(\mathbf{A})$. First, we have to prove that $h$ can be uniquely extended to a sequentially continuous D-homomorphisms on $\mathcal{M}\left(\mathbf{A}_{\sigma(\mathcal{X})}\right) \times$ $\mathcal{M}\left(\mathbf{A}_{\sigma(\mathcal{Y})}\right)$ into $\mathcal{M}(\mathbf{A})$. Let $Z$ be the disjoint union of $X$, and $Y$ and let $\mathcal{Z} \subseteq I^{Z}$ be the corresponding "disjoint union" of $\mathcal{X}$ and $\mathcal{Y}$. Then, we can identify $\mathcal{X} \times \mathcal{Y}$ and $\mathcal{Z}$, or more precisely, $\mathcal{M}\left(\mathbf{A}_{\sigma(\mathcal{X})}\right) \times \mathcal{M}\left(\mathbf{A}_{\sigma(\mathcal{Y})}\right)$ and $\mathcal{M}\left(\mathbf{A}_{\sigma(\mathcal{Z})}\right)$. Since $\mathcal{M}\left(\mathbf{A}_{\sigma(\mathcal{Z})}\right)$ is the epireflection of $\mathcal{Z}$, the assertion follows. Second, the embedding of $\mathcal{X} \times \mathcal{Y}$ into $\mathcal{M}\left(\mathbf{A}_{\sigma(\mathcal{X})}\right) \times \mathcal{M}\left(\mathbf{A}_{\sigma(\mathcal{Y})}\right)$ is an epimorphism, the proof is complete. 
Corollary 3.6. The epireflector sending objects of BID into CGBID is productive.

Corollary 3.7. Let $\mathcal{X} \subseteq I^{X}, \mathcal{Y} \subseteq I^{Y}$ be bold algebras and let $\mathcal{X} \times \mathcal{Y}$ be their product. Let $h$ be a sequentially continuous D-homomorphism on $\mathcal{X} \times \mathcal{Y}$ into $I$. Then $h$ can be uniquely extended to a sequentially continuous D-homomorphism on $\mathcal{M}\left(\mathbf{A}_{\sigma(\mathcal{X})}\right) \times \mathcal{M}\left(\mathbf{A}_{\sigma(\mathcal{Y})}\right)$ into $I$.

\section{Applications}

\subsection{IF-probability}

This subsection is devoted to relationships between IF-probability [31] -33 . and fuzzy probability [15]-[18, [28].

Let $X$ be a set. Recall that an IF-subset of $X$ is a pair $A=\left(\mu_{A}, \nu_{A}\right)$, where $\mu_{A}, \nu_{A}$ are fuzzy subsets of $X$ (called the membership and nonmembership functions of $A$, respectively) and $\mu_{A}+\nu_{A} \leq 1_{X}$, where $1_{X}$ denotes the constant function with value 1 . Clearly, for $\nu_{A}=1_{X}-\mu_{A},\left(\mu_{A}, \nu_{A}\right)$ can be considered as a fuzzy subset of $X\left(\nu_{A}=1_{X}-\mu_{A}\right.$ is a redundant information). Generalized random events in IF-probability are certain systems of IF-subsets.

Let $\mathbf{A}$ be a $\sigma$-field of subsets of a set $X$. Let $\mathcal{F}$ be the set of all measurable IF-subsets of $X$, i.e., pairs $\left(\mu_{A}, \nu_{A}\right)$ where $\mu_{A}, \nu_{A} \in \mathcal{M}(\mathbf{A}), \mu_{A}+\nu_{A} \leq 1_{X}$; we will call them IF-events. Let $A=\left(\mu_{A}, \nu_{A}\right)$ and $B=\left(\mu_{B}, \nu_{B}\right)$ be IF-events. B. R i e č a n (cf. [31]) defined two operations and a partial order on IF-events as follows:

$$
\begin{aligned}
& A \oplus B=\left(\left(\mu_{A}+\mu_{B}\right) \wedge 1_{X},\left(\nu_{A}+\nu_{B}-1_{X}\right) \vee 0_{X}\right) ; \\
& A \odot B=\left(\left(\mu_{A}+\mu_{B}-1_{X}\right) \vee 0_{X},\left(\nu_{A}+\nu_{B}\right) \wedge 1_{X}\right) ; \\
& A \preceq B \quad \text { if and only if }\left(\mu_{A} \leq \mu_{B}\right) \wedge\left(\nu_{B} \leq \nu_{A}\right) .
\end{aligned}
$$

Observe that IF-events are closed with respect to $\oplus$ and $\odot$, but not with respect to the operation $\neg$ defined by $\neg\left(\mu_{A}, \nu_{A}\right)=\left(1_{X}-\mu_{A}, 1_{X}-\nu_{A}\right)$. In [31], $\mathrm{R}$ i e $\check{c}$ a $\mathrm{n}$ defined states and observables for IF-events and succeeded in proving limit theorems for IF-probability (avoiding complement).

Denote

$$
A(\mathcal{M}(\mathbf{A}) \times \mathcal{M}(\mathbf{A}))=\{(u, v) \in \mathcal{M}(\mathbf{A}) \times \mathcal{M}(\mathbf{A}) ; u \leq v\} .
$$

Then, $A(\mathcal{M}(\mathbf{A}) \times \mathcal{M}(\mathbf{A}))$ is closed with respect to the binary Eukasiewicz operations, but it is not closed with respect to the complement $\neg$. Define a map $h$ of $\mathcal{F}$, the set of all measurable IF-subsets of $X$, into $A(\mathcal{M}(\mathbf{A}) \times \mathcal{M}(\mathbf{A}))$ by $h\left(\left(\mu_{A}, \nu_{A}\right)\right)=\left(\mu_{A}, 1_{X}-\nu_{A}\right)$. Then (cf. [31, [32]), $h$ is a bijection preserving the order and operations $\oplus, \odot$ in $\mathcal{F}$ and the usual order and the binary Eukasiewicz operations in $A(\mathcal{M}(\mathbf{A}) \times \mathcal{M}(\mathbf{A}))$. Accordingly, IF-events $\mathcal{F}$ can be viewed as the corresponding part $A(\mathcal{M}(\mathbf{A}) \times \mathcal{M}(\mathbf{A}))$ of the fuzzy probability domain $\mathcal{M}(\mathbf{A}) \times \mathcal{M}(\mathbf{A})$. In fact, $A(\mathcal{M}(\mathbf{A}) \times \mathcal{M}(\mathbf{A}))$ forms a "complement 
dense" part of $\mathcal{M}(\mathbf{A}) \times \mathcal{M}(\mathbf{A})$. Lemma 4.1 in 33 states that if $B$ is a bold algebra such that $A(\mathcal{M}(\mathbf{A}) \times \mathcal{M}(\mathbf{A})) \subseteq B \subseteq \mathcal{M}(\mathbf{A}) \times \mathcal{M}(\mathbf{A})$, then $B=\mathcal{M}(\mathbf{A}) \times \mathcal{M}(\mathbf{A})$. As proved by $\mathrm{R}$ i e č a $\mathrm{n}$ in 31, the system $\mathcal{F}$ of IF-events can be extended to an MV-algebra $\mathcal{M}$ in such a way that each state on $\mathcal{F}$ is the restriction of a uniquely determined state on $\mathcal{M}$. Moreover, $\mathrm{R}$ i e č a n proved a representation theorem for states on $\mathcal{M}$ (and hence on $\mathcal{F}$ ). The embedding of $A(\mathcal{M}(\mathbf{A}) \times \mathcal{M}(\mathbf{A})$ ) into $\mathcal{M}(\mathbf{A}) \times \mathcal{M}(\mathbf{A})$ is equivalent to the embedding of $\mathcal{F}$ into $\mathcal{M}$, but the representation theorem for states on $\mathcal{M}(\mathbf{A}) \times \mathcal{M}(\mathbf{A})$ is less technical and much more intuitive [32].

Let $\mathcal{X} \subseteq[0,1]^{X}$ be a bold algebra and let $\mathcal{M}\left(\mathbf{A}_{\sigma(\mathcal{X})}\right)$ be the corresponding generated Łukasiewicz tribe. Motivated by classical probability theory, we describe the transition from fuzzy events $(u, v) \in \mathcal{X} \times \mathcal{X}$ to fuzzy events in $\mathcal{M}\left(\mathbf{A}_{\sigma(\mathcal{X})}\right) \times \mathcal{M}\left(\mathbf{A}_{\sigma(\mathcal{X})}\right)$. Observe that the transition from fields of sets to the generated $\sigma$-fields enables us to exploit many powerful tools of mathematical analysis in probability theory.

Let $\mathcal{X} \subseteq[0,1]^{X}$ be a bold algebra. Put $A(\mathcal{X} \times \mathcal{X})=\{(u, v) \in \mathcal{X} \times \mathcal{X} ; u \leq v\}$. Clearly, $A(\mathcal{X} \times \mathcal{X})$ is closed with respect to the Eukasiewicz operations $\oplus, \odot$.

Now, let us turn to the transition from $A(\mathcal{X} \times \mathcal{X})$ to $\mathcal{X} \times \mathcal{X}$, resp. from $A\left(\mathcal{M}\left(\mathbf{A}_{\sigma(\mathcal{X})}\right) \times \mathcal{M}\left(\mathbf{A}_{\sigma(\mathcal{X})}\right)\right)$ to $\mathcal{M}\left(\mathbf{A}_{\sigma(\mathcal{X})}\right) \times \mathcal{M}\left(\mathbf{A}_{\sigma(\mathcal{X})}\right)$. Note that if $\mathbf{A}$ is a field of sets considered as a bold algebra $\mathcal{X}$, then $\mathcal{M}(\sigma(\mathbf{A}))$ is equal to $\mathcal{M}\left(\mathbf{A}_{\sigma(\mathcal{X})}\right)$.

The next lemma can be proved analogously as in [33, Lemma 4.1].

Lemma 4.1. Let $B$ be a bold algebra such that $A(\mathcal{X} \times \mathcal{X}) \subseteq B \subseteq \mathcal{X} \times \mathcal{X}$. Then, $B=\mathcal{X} \times \mathcal{X}$.

Having in mind the relationships between $I F$ probability and fuzzy probability, consider the following question. Are there two isomorphic categories such that the objects of the first are of the form $A(\mathcal{X} \times \mathcal{X})$ and the objects of the second are of the form $\mathcal{X} \times \mathcal{X}$, where $\mathcal{X}$ is a bold algebra?

Let $\mathcal{X} \subseteq I^{X}, \mathcal{Y} \subseteq I^{Y}$ be bold algebras and let $h$ be a sequentially continuous D-homomorphism of $\mathcal{X}$ into $\mathcal{Y}$. For $(u, v) \in \mathcal{X} \times \mathcal{X}$, define $\bar{h}(u, v)=(h(u), h(v))$. Denote $\bar{h}$ the resulting map of $\mathcal{X} \times \mathcal{X}$ into $\mathcal{Y} \times \mathcal{Y}$. Denote $\bar{h}_{A}$ the restriction of $\bar{h}$ to $A(\mathcal{X} \times \mathcal{X})$.

\section{LEMMA 4.2.}

(i) $\bar{h}$ a sequentially continuous D-homomorphism of $\mathcal{X} \times \mathcal{X}$ into $\mathcal{Y} \times \mathcal{Y}$,

(ii) $\bar{h}(u, v) \in A(\mathcal{Y} \times \mathcal{Y})$ whenever $(u, v) \in A(\mathcal{X} \times \mathcal{X})$.

P r o of. Both assertions follow from straightforward calculations.

The preceding arguments lead to the following category AIF:

(i) the objects are systems $A(\mathcal{X} \times \mathcal{X})$, where $\mathcal{X}$ is a bold algebra, equipped with the natural partial order inherited from $\mathcal{X} \times \mathcal{X}$ and two Eukasiewicz operations $\oplus, \odot$, inherited from $\mathcal{X} \times \mathcal{X}$; 
(ii) the morphisms are of the form $\bar{h}_{A}$, where $h$ is a sequentially continuous D-homomorphism of the bold algebra $\mathcal{X}$ into the bold algebra $\mathcal{Y}$.

Denote $\mathbf{B I D}^{2}$ the following subcategory of $\mathbf{B I D}_{2}$ :

(i) the objects are bold algebras of the form $\mathcal{X} \times \mathcal{X}$;

(ii) the morphisms are of the form $\bar{h}$, where $h$ is a sequentially continuous D-homomorphism of a bold algebra $\mathcal{X}$ into a bold algebra $\mathcal{Y}$.

Theorem 4.3. The categories $\mathbf{A I F}$ and $\mathbf{B I D}^{2}$ are isomorphic.

P r o of. It follows from the previous two lemmas that there is a one-to-one correspondence between the objects of $\mathbf{A I F}$ and $\mathbf{B I D}^{2}$ and the morphisms of $\mathbf{A I F}$ and $\mathbf{B I D}^{2}$, respectively, assigning $\mathcal{X} \times \mathcal{X}$ to $A(\mathcal{X} \times \mathcal{X})$ and $\bar{h}$ to $\bar{h}_{A}$, respectively. Clearly, this yields an isomorphism functor.

Conclusion. Let $\mathcal{X} \subseteq I^{X}$ be a bold algebra and let $\mathcal{X} \times \mathcal{X}$ be its power. Then (cf. Theorem 3.4), states on $\mathcal{X} \times \mathcal{X}$ are exactly convex combinations of states on $\mathcal{X}$. In view of Theorem 4.3, IF-probability can be interpreted within fuzzy probability theory.

\subsection{Fuzzy probability}

Fuzzy probability theory [34, [18] generalizes the classical one. More information can be found in [8], [17] and references therein.

The results of Section 3 and Section 4.1 indicate that it might be interesting to develop generalized probability theories based on the categories $\mathbf{B I D}^{2}$ and $\mathbf{B I D}_{2}$. In the first case, the resulting generalization could provide a technical support to IF-probability. In the second case, the resulting generalization could lead to a ramification of fuzzy probability.

\section{REFERENCES}

[1] ADÁMEK, J.: Theory of Mathematical Structures. Reidel, Dordrecht, 1983.

[2] CHOVANEC, F.-KÔPKA, F.: D-posets, in: Handbook of Quantum Logic and Quantum Structures: Quantum Structures. (K. Engesser at al., eds.), Elsevier, Amsterdam, 2007, pp. 367-428.

[3] DVUREČENSKIJ, A.-PULMANNOVÁ, S.: New Trends in Quantum Structures. Kluwer Academic Publ., Dordrecht; Ister Science, Bratislava, 2000.

[4] FRIČ, R.: Remarks on sequential envelopes, Rend. Istit. Math. Univ. Trieste 26 (1988), 604-612.

[5] FRIČ, R.: Stone-type duality and its applications to probability, Topology Proc. 22 (Summer 1997), 125-137.

[6] FRIČ, R.: Eukasiewicz tribes are absolutely sequentially closed bold algebras, Czechoslovak Math. J. 52 (2002), 861-874.

[7] FRIČ, R.: Duality for generalized events, Math. Slovaca 54 (2004), 49-60. 


\section{REAL FUNCTIONS AND EXTENSION OF GENERALIZED PROBABILITY MEASURES II}

[8] FRIČ, R.: Remarks on statistical maps and fuzzy (operational) random variables. Tatra Mt. Math. Publ. 30 (2005), 21-34.

[9] FRIČ, R.: Extension of measures: a categorical approach, Math. Bohemica 130 (2005), 397-407.

[10] FRIČ, R.: Measures: continuity, measurability, duality, extension, Tatra Mt. Math. Publ. 42 (2009), 161-174.

[11] FRIČ, R.: Extension of domains of states, Soft Comput. 13 (2009), 63-70.

[12] FRIČ, R.: From probability to sequences and back, Rend. Istit. Mat. Univ. Trieste 44 (2012), 285-296.

[13] FRIČ, R.: On D-posets of fuzzy sets, Math. Slovaca 64 (2014), 545-554.

[14] FRIČ, R.-PAPČO, M.: On probability domains, Internat. J. Theoret. Phys. 49 (2010), 3092-3100.

[15] FRIČ, R.-PAPČO, M.: A categorical approach to probability theory, Studia Logica 94 (2010), 215-230.

[16] FRIČ, R.-PAPČO, M.: On probability domains II, Internat. J. Theoret. Phys. 50 (2011), 3778-3786.

[17] FRIČ, R.-PAPČO, M.: On probability domains III, Internat. J. Theoret. Phys. (to appear).

[18] GUDDER, S.: Fuzzy probability theory, Demonstratio Math. 31 (1998), 235-254.

[19] HAVLÍČKOVÁ, J.: Real functions and the extension of generalized probability measure, Tatra Mt. Math. Publ. 55 (2013), 85-94.

[20] KÔPKA, F.-CHOVANEC, F.: D-posets, Math. Slovaca 44 (1994), 21-34.

[21] JUREČKOVÁ, M.: The measure extension theorem for $M V$-algebras, Tatra Mt. Math. Publ. 6 (1995), 56-61.

[22] NOVÁK, J.: Ueber die eindeutigen stetigen Erweiterungen stetiger Funktionen, Czechoslovak Math. J. 8 (1958), 344-355.

[23] NOVÁK, J.: On the sequential envelope, in: General Topology and its Relations to Modern Analysis and Algeba, Proc. of the 1st Prague Topological Symp., 1961, Publ. House of the Czechoslovak Academy of Sciences, Prague, 1962, pp. 292-294.

[24] NOVÁK, J.: On convergence spaces and their sequential envelopes, Czechoslovak Math. J. 15 (1965), 74-100.

[25] NOVÁK, J.: On sequential envelopes defined by means of certain classes of functions, Czechoslovak Math. J. 18 (1968), 450-456.

[26] PAPČO, M.: On measurable spaces and measurable maps, Tatra Mt. Math. Publ. 28 (2004), 125-140.

[27] PAPČO, M.: On effect algebras, Soft Comput. 12 (2007), 26-35.

[28] PAPČO, M.: Fuzzification of probabilistic objects, in: 8th Conference of the European Society for Fuzzy Logic and Technology_EUSFLAT '13 (G. Pasi at al., eds.), Milano, Italy, 2013, Atlantis Press, Amsterdam, 2013, pp. 67-71.

[29] RIEČAN, B.-MUNDICI, D.: Probability on MV-algebras, in: Handbook of Measure Theory, Vol. I and II (E. Pap, ed.), North-Holland, Amsterdam, 2002, pp. 869-910.

[30] RIEČAN, B.-NEUBRUNN, T.: Integral, Measure, and Ordering. Kluwer Acad. Publ., Dordrecht, 1997.

[31] RIEČAN, B.: Analysis of fuzzy logic models, in: Intelligent Systems (V. M. Koleshko, ed.), InTech, Rijeka, 2012, pp. 219-244.

[32] SKR̆IVÁNEK, V.: Real functions in generalized probability (submitted). 


\section{JANA HAVLÍČKOVÁ}

[33] SKŘIVÁNEK, V.-FRIČ, R.: Generalized random events, Internat. J. Theoret. Phys. (to appear).

[34] ZADEH, L. A.: Probability measures of fuzzy events, J. Math. Anal. Appl. 23 (1968), 421-427.

Received February 21, 2015

Faculty of Mathematics,

Physics and Informatics

Comenius University

Mlynská dolina

SK-842-48 Bratislava

SLOVAKIA

E-mail: jani.havlickova@gmail.com 